\title{
ON THE ITERATION OF TRANSFORMATIONS IN NONCOMPACT MINIMAL DYNAMICAL SYSTEMS
}

\author{
FELIX E. BROWDER
}

Let $A$ be a Hausdorff space, $\phi$ a continuous mapping of $A$ into itself. It is the purpose of the present paper to discuss various topics centering around the following question: If $g$ is a bounded continuous function on $A$, does there exist a bounded continuous function $f$ on $A$ such that $f(\phi a)-f(a)=g(a)$ for all $a$ in $A$ ? Suppose that for each $a_{0}$ in $A$, the set $\left\{\phi^{n} a_{0}, n \geqq 0\right\}$ is dense in $A$. Theorem 1 asserts that a necessary and sufficient condition for the existence of such an $f$ is that $\left|\sum_{k=0}^{j} g\left(\phi^{k} a\right)\right|$ should be uniformly bounded for all positive $j$ and all points $a$ of $A$. For homeomorphisms of compact spaces, this result was previously obtained by Gottschalk and Hedlund [5, Theorem 14.11 , p. 135]. ${ }^{1}$

A related problem for linear operators in a Banach space is obtained by letting $X$ be the Banach space of bounded continuous functions on $A$ with the uniform norm, $T$ the linear transformation of $X$ into itself defined by $(T f)(a)=f(\phi a), a \in A$. In terms of $X$ and $T$, Theorem 1 states that $g$ will lie in the range of $(I-T)$ if and only if the sequence of norms $\left\|\sum_{k=0}^{j} T^{k} g\right\|$ is uniformly bounded for all positive $j$. In a reflexive Banach space, this characterization of the range of $(I-T)$ is valid for any linear transformation $T$ for which $\left\|T^{n}\right\|$ is bounded for all $n$. A sufficient condition in a general Banach space would seem to require an assumption that the elements $\left\{\sum_{k=0}^{j} T^{k} g\right\}$ lie for all $j$ in a fixed weakly compact subset $K$ of $X$. It would be interesting to obtain a proof of Theorem 1 along these lines. We shall content ourselves with showing by these methods that if $m$ is a totally-finite measure on a $\sigma$-algebra on $A, L^{\infty}(m)$ the space of $m$-essentially bounded measurable functions, $\phi$ a measure preserving mapping of $A$ into $A$, then in order that for an element $g$ in $L^{\infty}(m)$, there should exist an $f$ in $L^{\infty}(m)$ such that $f(\phi a)-f(a)=g(a)$ a.e. in $m$, it is necessary and sufficient that $m$-ess. sup. $\left|\sum_{k=0}^{j} g\left(\phi^{k} a\right)\right|$ should be uniformly bounded for all positive $j .^{2}$

Such a result raises another sort of question. For a topological space $A$ if $g$ is continuous and $f$ is a solution of the equation $f(\phi a)$

Received by the editors May 24, 1957.

1 The writer's attention was drawn to this question by reading a preprint of [3] in which a theorem of this type is proved for rotations of the circle. This case was already treated by Hedlund in $[6$, Theorem 3.1, p. 557].

${ }^{2}$ A result in $L^{\infty}$ for rotations of the circle was already proved by J. Wermer. (Cf. footnote, p. 557 of [6].) 
$-f(a)=g(a)$, with $f$ lying in some larger class of functions, must $f$ be necessarily continuous after change on some negligible set? We place the question in a more definite setting. Let $A_{1}$ be a Hausdorff space, $A_{2}$ a compact topological group, $\phi$ a homeomorphism of $A_{1}$ onto itself such that $A_{1}$ is a minimal orbit closure under $\phi, \psi_{0}$ a continuous map of $A_{1}$ into $A_{2}$. Suppose there exists a Baire function $h$ from $A_{1}$ to $A_{2}$ satisfying the relation

$$
h\left(\phi a_{1}\right)=\psi_{0}\left(a_{1}\right) \cdot h\left(a_{1}\right),(\cdot, \text { the group product }),
$$

for all $a_{1}$ outside some set of the first category in $A_{1}$. Then if $A_{1}$ is a Baire space, $h$ is continuous after change on a set of the first category.

A similar result is valid if $A_{2}$ is merely a compact space, $\psi_{0}$ a homeomorphism of $A_{2}$ onto itself which generates an equicontinuous transformation group of $A_{2}$, and (1) is replaced by

$$
h\left(\phi a_{1}\right)=\psi_{0}\left(h a_{1}\right) \text {. }
$$

In this form, the result has been established by S. Kakutani in [7] by rather different methods. One interesting feature of the present proof is that it is valid also under the following hypotheses: $A_{1}$ a measure space with a measure $m$ such that all open sets are measurable and have positive measure, $\phi$ maps null sets on null sets, $h$ a function from $A_{1}$ to $A_{2}$ continuous on the complement of a set of zero measure. Then $h$ is continuous on the whole of $A_{1}$ after replacement on a set of measure zero. An extension is given for functional equations of a more general type than (1)'.

1: Let $A$ be a Hausdorff space, $\phi$ a continuous mapping of $A$ into itself. We assume that $A$ is a minimal orbit closure under $\phi$, i.e., for every $a_{0}$ in $A$, the closure of the set $\left\{\phi^{n} a_{0}, n \geqq 0\right\}$ coincides with $A$. Let $B$ be another Hausdorff space, $\psi$ a continuous mapping of the Cartesian product $A \times B$ into $B$.

We define a continuous mapping $\pi$ of $A \times B$ into itself by setting $\pi(a, b)=(\phi a, \psi(a, b))$. If $\pi^{n}$ is the $n$th iterate of $\pi, 0(a, b)$ is the orbit of $(a, b)$ under the mapping $\pi$, i.e. $O(a, b)=\bigcup_{n_{2} 0}\left\{\pi^{n}(a, b)\right\}$, then we let $F(a, b)$ be the closure of $O(a, b)$ in $A \times B$. Let $p_{A}$ and $p_{B}$ be the projection mappings of $A \times B$ on its first and second components respectively, $p_{A}(a, b)=a, p_{B}(a, b)=b$. We shall assume in the following that for each point $(a, b)$ in $A \times B, p_{B}(F(a, b))$ is contained in a compact subset of $B$.

Consider the family $J$ of subsets of $A \times B$, where $J=\{F \mid F$ is a nonempty closed subset of $A \times B ;(a, b) \in F$ implies that $\pi(a, b) \in F$; $p_{B}(F)$ is contained in a compact subset of $\left.B\right\}$. Since for any point 
$\left(a_{0}, b_{0}\right)$ in $A \times B, F\left(a_{0}, b_{0}\right)$ is an element of $J, J$ is certainly not vacuous.

Lemma 1. If $F \in J$, then $p_{A}(F)=A$.

Proof. Let $\left(a_{0}, b_{0}\right)$ be a point of $F$. Since $\pi^{n}\left(a_{0}, b_{0}\right) \in F, p_{A} \pi^{n}\left(a_{0}, b_{0}\right)$ $\in p_{A}(F)$. Thus $p_{A}(F)$ contains the dense set $\left\{\phi^{n} a_{0}\right\}$ and therefore is dense in $A$. On the other hand, $F$ is closed in $A \times B, F \subset A$ $\times \mathrm{Cl}\left(p_{B}(F)\right)$, and $\mathrm{Cl}\left(p_{B}(F)\right)$ is compact in $B$. Therefore, $p_{A}(F)$ is closed in $A\left[1\right.$, Exercise 8, p. 68]. Since $p_{A}(F)$ is dense and closed in $A, p_{A}(F)=A$.

Lemma 2. $J$ has a minimal element under inclusion. Every orbit closure $F(a, b)$ contains a minimal element of $J$.

Proof. By the Lemma of Zorn, it suffices to prove that every subfamily of $J$ which is linearly ordered with respect to inclusion has a lower bound in $J$. Let $L=\left\{F_{\alpha}\right\}$ be such a family. Then $F_{0}=\bigcap_{\alpha} F_{\alpha}$ is a closed invariant set under $\pi$ while $p_{B}\left(F_{0}\right)$ is certainly contained in a compact subset of $B$. To prove that $F_{0} \in J$, we must show $F_{0} \neq \phi$. Let $a_{0}$ be a point of $A, G_{\alpha}=F_{\alpha} \cap p_{A}^{-1}\left(a_{0}\right)$. By Lemma $1, G_{\alpha}$ is a family of closed sets in $A \times B$ such that every finite subfamily has a nonempty intersection. Moreover, each $G_{\alpha}$ is a closed subset of $a_{0} \times \mathrm{Cl}\left(p_{B}\left(F_{\alpha}\right)\right)$, which is compact since it is mapped homeomorphically by $p_{B}$ on the compact set $\mathrm{Cl}\left(p_{B}\left(F_{\alpha}\right)\right)$. Since all the $G_{\alpha}$ are compact, $G_{0}=\bigcap_{\alpha} G_{\alpha}$, is nonempty, and, since $G_{0} \subset F_{0}, F_{0}$ is nonempty.

Let $\zeta$ be a homeomorphism of $B$ onto itself commuting with $\psi$, i.e. such that $\psi(a, \zeta b)=\zeta \psi(a, b)$ for all $a \in A, b \in B$. Let $S_{\zeta}$ be the homeomorphism of $A \times B$ onto itself defined by $S_{\zeta}(a, b)=(a, \zeta b)$.

Lemma 3. Let $F_{0}$ be a minimal element of $J$ and suppose that for a fixed point $a$ in $A$, the points $(a, b)$ and $\left(a, b_{1}\right)$ lie in $F$. Suppose further that there exists a homeomorphism $\zeta$ of $B$ onto $B$ commuting with $\psi$, such that $\zeta b=b_{1}$. Then $S_{\zeta} F_{0}=F_{0}$.

Proof. From the fact that $\zeta$ commutes with $\psi$, we see that $S_{\zeta} \pi(a, b)$ $=(\phi a, \zeta \psi(a, b))=(\phi a, \psi(a, \zeta b))=\pi S_{\zeta}(a, b)$. Thus $S_{\zeta} \pi^{n}=\pi^{n} S_{\zeta}$, and $S_{\zeta}(O(a, b))=O(a, \zeta b)$. Since $S_{\zeta}$ is a homeomorphism, $S_{\zeta} F(a, b)$ $=F(a, \zeta b)$. Since $F_{0}$ is a minimal element of $J, F_{0}=F(a, b)=F\left(a, b_{1}\right)$. But $S_{\zeta} F_{0}=S_{\zeta} F(a, b)=F(a, \zeta b)=F\left(a, b_{1}\right)=F_{0}$.

THEOREM 1. Let $\phi$ be a continuous mapping of the Hausdorff space $A$ into itself with $A$ a minimal orbit closure under $\phi$. Let $g$ be a bounded continuous function from $A$ to the $n$-dimensional Euclidean space $R^{n}$. In order that there should exist a bounded continuous function $f$ from $A$ to $R^{n}$ such that $f(\phi a)-f(a)=g(a)$ for all $a$ in $A$, it is necessary and 
sufficient that there exist a constant $M>0$ with

$$
\sup _{a \in A}\left|\sum_{k=0}^{j} g\left(\phi^{k} a\right)\right| \leqq M \text { for all } j \geqq 0 .
$$

Proof of Theorem 1. Necessity is obvious for if $g(a)=f(\phi a)$ $-f(a)$, then $\left|\sum_{k=0}^{j} g\left(\phi^{k} a\right)\right|=\left|f\left(\phi^{i+1} a\right)-f(a)\right| \leqq 2 \sup |f(a)|$.

To prove sufficiency, we specialize our preceding discussion by taking $B=R^{n}$ and setting $\psi(a, r)=r+g(a)$ for $a \in A, r \in R^{n}$. The corresponding mapping $\pi$ is defined by $\pi(a, r)=(\phi a, r+g(a))$. The condition (2) is equivalent to the fact that the orbit of any point $(a, r)$ under $\pi$ has a bounded and hence precompact image in $R^{n}$ under the projection map $p_{R^{n}}$ of $A \times R^{n}$ into $R^{n}$. Hence the conclusions of Lemmas 1,2 , and 3 are valid for this mapping $\pi$. Let $F_{0}$ be a minimal closed invariant set in $A \times R^{n}$ with respect to $\pi$. Suppose that for some point $a$ in $A, p_{A}^{-1}(a) \cap F_{0}$ contained two distinct points $(a, r)$, $\left(a, r_{1}\right)$. Let $\xi=r-r_{1}, \zeta_{\xi}$ the homeomorphism of $R^{n}$ onto itself defined by $\zeta_{\xi}(r)=r+\xi$. Then $\zeta_{\xi}$ commutes with $\psi, \zeta_{\xi}\left(r_{1}\right)=r$, and Lemma 3 is applicable. Thus if $S_{\zeta_{\xi}}(a, r)=(a, r+\xi), S_{\zeta_{\xi}} F_{0}=F_{0}$. But then $S_{\zeta \xi}^{m} F_{0}=F_{0}$ for any positive integer $m$, contradicting the boundedness of the second component for elements of $F_{0}$. Thereby, we have shown that $F_{0}$ has at most one point $(a, r)$ for a given $a \in A$.

Let $f$ be the function from $A$ to $R^{n}$ defined uniquely by the condition $(a, f(a)) \in F_{0}$. By Lemma $1, f$ is defined on all of $A . f$ can be considered as a function from $A$ to the compact set $\mathrm{Cl}\left(p_{R^{n}} F_{0}\right)$. Since $F_{0}$, the graph of $f$, is closed, $f$ is continuous [1, Exercise 12, p. 68]. Since $\pi(a, f(a)) \in F_{0}$, we have $(\phi a, f(a)+g(a)) \in F_{0}$, i.e. $f(\phi a)=f(a)$ $+g(a)$.

REMARK. Following a remark of Kakutani, we note that the existence of a minimal subset in $J$ under the hypotheses of Theorem 1 can be proved in an elementary way without the use of Zorn's Lemma or the Axiom of Choice. Let $F_{0}=F\left(a_{0}, r_{0}\right)$ for a fixed element $\left(a_{0}, r_{0}\right)$ in $A \times R^{n}$. We shall show that $F_{0}$ is a minimal element of $J$. It suffices to show that if $(a, r) \in F_{0}$, then $\left(a_{0}, r_{0}\right) \in F(a, r)$. We note first that if $\left(a_{0}, r_{1}\right) \in F_{0}$, and $\xi=r_{1}-r_{0}$, then $S_{\xi_{\xi}} F_{0}=S_{\zeta_{\xi}} F\left(a_{0}, r_{0}\right)$ $=F\left(a_{0}, r_{1}\right) \subset F_{0}$. If $\xi \neq 0$, then $S_{\xi \xi}^{m} F_{0} \subset F_{0}$ for all $m>0$, contradicting (2). Thus $\xi=0$ and $\left(a_{0}, r_{0}\right)$ is the only point in $p_{A}^{-1}\left(a_{0}\right) \cap F_{0}$. But $p_{A}^{-1}\left(a_{0}\right) \cap F(a, r)$ is contained in $p_{A}^{-1}\left(a_{0}\right) \cap F_{0}$ and is nonempty by Lemma 1. It follows that $\left(a_{0}, r_{0}\right) \in F(a, r)$ and $F_{0}$ is minimal.

2. Let $X$ be a Banach space, $T$ a continuous linear transformation of $X$ into itself.

Lemma 4. A sufficient condition for $g$ in $X$ to lie in the range of 
$(I-T)$ is that the set of elemenets $\left\{\sum_{k=0}^{j} T^{k} g\right\}$ should lie for $j \geqq 0$ in a fixed weakly compact subset $K$ of $X$.

Proof. By theorems of Eberlein and M. Krein (cf. [4]), the convex closure $K^{\prime}$ of $K \cup\{0\}$ is weakly sequentially compact. By the principle of uniform boundedness, there exists $M>0$ such that

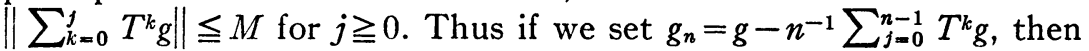
$g_{n}$ will converge strongly to $g$ as $n \rightarrow \infty$. Furthermore, each $g_{n}$ lies in the range of $(I-T)$ since $g_{n}=(I-T)\left\{n^{-1} \sum_{k=1}^{n-1}\left(\sum_{j=0}^{k-1} T^{i} g\right)\right\}$. Let us set $h_{k}=\sum_{j=0}^{k-1} T^{j} g, f_{n}=\left\{\sum_{k=1}^{n-1} h_{j}\right\} \cdot n^{-1}$. Then $g_{n}=(I-T) f_{n}$, while the $f_{n}$ lie for all $n$ in the weakly sequentially compact set $K^{\prime}$. Choose a subsequence $f_{n_{i}}$ converging weakly to an element $f$ of $X$ as $i \rightarrow \infty$. Then $(I-T) f_{n_{i}}$ converges weakly to $(I-T) f$. But $g_{n_{i}}=(I-T) f_{n_{i}}$ converges strongly to $g$. Hence $g=(I-T) f$.

Lemma 5. Let $X$ be a reflexive Banach space, $T$ a continuous linear transformation of $X$ into itself. A sufficient condition that $\mathrm{g}$ lie in the range of $(I-T)$ is that $\left\|\sum_{k=0}^{j} T^{k} g\right\|$ be uniformly bounded for $j \geqq 0$. If $\left\|T^{n}\right\| \leqq M^{\prime}$ for $n \geqq 0$, the condition is also necessary.

Proof. The necessity is obvious, since if $g=(I-T) f,\left\|\sum_{k=0}^{j} T^{k} g\right\|$ $=\left\|f-T^{i+1} f\right\| \leqq 2 M^{\prime}$. Sufficiency follows from Lemma 4 since every closed ball about zero in a reflexive space is weakly compact.

Theorem 2. Let $A$ be a measure space with a totally finite measure $m, \phi$ a measure preserving mapping of $A$ into $A$. In order that for a function $g$ in $L^{\infty}(m)$, there should exist an $f \in L^{\infty}(m)$ such that $f(\phi a)$ $-f(a)=g(a)$ a.e. in $m$, it is necessary and sufficient that

$$
m \text {-ess. sup. }\left|\sum_{k=0}^{j} g\left(\phi^{k} a\right)\right|
$$

should be uniformly bounded for $j \geqq 0$.

Proof of Theorem 2. Choose a value of $p, 1<p<\infty$. Let $T$ mapping $L^{p}(m)$ into itself be defined by $(T f)(a)=f(\phi a), a \in A$. Then

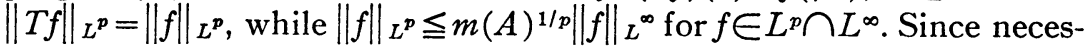
sity is obvious, we consider only sufficiency. Let $g$ be our given func-

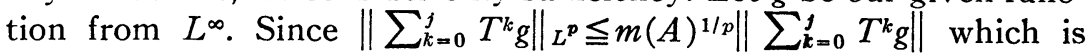
uniformly bounded for $j \geqq 0$, applying Lemma 5 to the reflexive space $L^{p}(m)$, we conclude that there exists $f_{0} \in L^{p}(m)$ such that $f_{0}(\phi a)$ $-f_{0}(a)=g(a)$. Since the mean ergodic theorem holds for $T$ in the reflexive space $L^{p}(m)$, [8] the ergodic means $n^{-1} \sum_{j=0}^{n-1} T^{j} f_{0}$ converges to an element $f_{1}$ of $L^{p}(m)$ in the strong topology of $L^{p}(m)$ and $(I-T) f_{1}=0$. Let $f=f_{0}-f_{1}$. Then $f(\phi a)-f(a)=g(a)$ a.e. while $n^{-1} \sum_{j=0}^{n-1} T^{i} f \rightarrow 0$ in $L^{p}(m)$ as $n \rightarrow \infty$. Set $h_{n}=-n^{-1} \sum_{j=1}^{n} \sum_{k=0}^{j-1} T^{k} g$. 
Then $\left\|h_{n}\right\|_{L^{\infty}}$ are uniformly bounded while $h_{n}=f-n^{-1} \sum_{j=1}^{n} T^{i f}$ converges in $L^{p}(m)$ to $f$ as $n \rightarrow \infty$. Choosing a subsequence which converges to $f$ a.e., it follows that $f \in L^{\infty}(m)$.

3. Let $A_{1}$ and $A_{2}$ be two Hausdorff spaces, with $A_{1}$ a Baire space, i.e. of the second category on itself. Let $\phi$ be a homeomorphism of $A_{1}$ onto itself such that $A_{1}$ is a minimal orbit closure under $\phi$. Let $\psi$ be a continuous mapping from $A_{1} \times A_{2}$ into $A_{2}$. We shall consider functions $h$ from $A_{1}$ to $A_{2}$ which satisfy the condition

$$
h\left(a_{1}\right)=\psi\left(a_{1}, h\left(a_{1}\right)\right), \quad a_{1} \in A_{1} .
$$

The function $h$ will be said to be a Baire function if there exists a set $S$ of the first category in $A_{1}$ such that $h$ is a continuous mapping of $A_{1}-S$ into $A_{2}$. If $A_{2}$ is a metric space, this definition includes all functions obtained by a sequence of pointwise sequential limits starting with continuous functions [2, Exercise 14, p. 81].

A family $H$ of homeomorphisms of $A_{2}$ is said to be universally transitive if for every pair of distinct points $a_{2}, a_{2}^{\prime}$ in $A_{2}$ there is a $\zeta$ in $H$ such that $\zeta a_{2}=a_{2}^{\prime}$.

Theorem 3. Let $h$ be a Baire function from $A_{1}$ to $A_{2}$ for which (3) holds outside some set $S_{1}$ of first category in $A_{1}$. Suppose that $A_{2}$ is compact and that there exists a universally transitive family II of homeomorphisms of $A_{2}$, each of which has no fixed points and commutes with $\psi$. Then after change on a set of the first category in $A_{1}, h$ can be made into a continuous function from $A_{1}$ to $A_{2}$ satisfying (3) for all $a_{1}$ in $A_{1}$.

Proof. Let $S_{0}=\cup_{n \geqq 0}\left\{\phi^{n}(S) \cup \phi^{n}\left(S_{1}\right)\right\}$. Since $\phi$ is a homeomorphism, $S_{0}$ is of first category in $A_{1} . A_{1}-S_{0}$ is an invariant set with respect to $\phi$ and dense in $A_{1}, h$ is continuous from $A_{1}-S_{0}$ to $A_{2}$, and (3) holds for all $a_{1}$ in $A_{1}-S_{0}$. If we set $B=A_{2}$ in the discussion of $\S 1$ and $\pi\left(a_{1}, a_{2}\right)=\left(\phi a_{1}, \psi\left(a_{1}, a_{2}\right)\right)$, the results of Lemmas 1,2 , and 3 are valid for $\pi$. Let $a_{1}^{\prime}$ be a point of $A_{1}-S_{0}, a_{2}^{\prime}=h\left(a_{1}^{\prime}\right), F_{0}$ a minimal invariant set contained in $F\left(a_{1}^{\prime}, a_{2}^{\prime}\right)$. The condition (3) on $h$ in $A_{1}-S_{0}$ implies since $h$ is continuous on $A_{1}-S_{0}$, that if $G$ is the graph of $h$ on $A_{1}-S_{0}$, then $G=F\left(a_{1}^{\prime}, a_{2}^{\prime}\right) \cap p_{A_{1}}^{-1}\left(A_{1}-S_{0}\right)$. We shall show that $F_{0}=F\left(a_{1}^{\prime}, a_{2}^{\prime}\right)$ and that for each $a_{1}$ in $A_{1}, p_{A_{1}}^{1}\left(a_{1}\right) \cap F_{0}$ consists of a single point. The function $f$ whose graph is $F_{0}$ will then be the desired continuous extension of $h$.

It suffices to show that if $\left(a_{1}, a_{2}\right)$ and $\left(a_{1}, a_{2}^{*}\right)$ lie in $F_{0}$, then $a_{2}=a_{2}^{*}$. If not, there is a homeomorphism $\zeta \in H$ commuting with $\psi$ and without fixed points on $A_{2}$ such that $\zeta a_{2}=a_{2}^{*}$. By Lenıma 3 , however $S_{\zeta} F_{0}=F_{0}$. Since $\zeta$ has no fixed points, $S_{\zeta}$ has no fixed points. But then $F_{0}$ and a fortiori $F\left(a_{1}^{\prime}, a_{2}^{\prime}\right)$ would have at least two points over 
every point of $A_{1}$. Since over the points of $A_{1}-S_{0}$, it has only one point, this is impossible.

We may specialize Theorem 3 in two ways: (1) by letting $A_{2}$ be a compact group, $\psi_{0}$ a mapping of $A_{1}$ into $A_{2}, \psi\left(a_{1}, a_{2}\right)=\psi_{0}\left(a_{1}\right) \cdot a_{2}$, the homeomorphism family $H$ be the elements of $A_{2}-\{e\}$ acting by right multiplication on $A_{2} ;(2)$ by letting $A_{2}$ be a compact space, $\psi_{0}$ be a homeomorphism of $A_{2}$ onto itself such that the group generated by $\psi_{0}$ is equicontinuous, $\psi\left(a_{1}, a_{2}\right)=\psi_{0}\left(a_{2}\right), H$ the closure of the group of homeomorphisms generated by $\psi_{0}$ except for the identity. In this second case we may replace $A_{2}$ by the orbit closure under $\psi_{0}$ of one of the values taken by $h$ on an element of $A_{1}-S_{0}$. It is known [5, 9.33 , pp. 78-79] that on this orbit closure $H$ is universally transitive and, unless the orbit closure is finite, the elements of $H$ have no fixed points on this set. If we modify $h$ to make it a continuous mapping into this set, it will be a continuous mapping into $A_{2}$.

In these two cases the specialized forms of Theorem 3 become:

Theorem 4. Let $A_{1}$ be a Baire space, $A_{2}$ a compact group, $\phi$ a homeomorphism of $A_{1}$ onto itself such that $A_{1}$ is a minimal orbit closure under $\phi$. Let $\psi_{0}$ be a continuous mapping of $A_{1}$ into $A_{2}$. Suppose that the Baire function $h$ satisfies the relation

$$
h\left(\phi a_{1}\right)=\psi_{0}\left(a_{1}\right) \cdot h\left(a_{1}\right)
$$

for all $a_{1}$ outside a set of the first category in $A_{1}$. Then after change on a set of the first category in $A_{1}, h$ can be made into a continuous function from $A_{1}$ to $A_{2}$ which satisfies (1) for all $a_{1} \in A_{1}$.

Theorem 5. Let $A_{1}$ be a Baire space, $A_{2}$ a compact space, $\phi$ a homeomorphism of $A_{1}$ onto itself under which $A_{1}$ is a minimal orbit closure, $\psi_{0}$ a homeomorphism of $A_{2}$ into itself which generates an equicontinuous group of homeomorphisms of $A_{2}$. Suppose that the Baire function $h$ from $A_{1}$ to $A_{2}$ satisfies the relation

$$
h\left(\phi a_{1}\right)=\psi_{0}\left(h a_{1}\right)
$$

for all $a_{1}$ outside a set of the first category in $A_{1}$. Then after change on $a$ set of the first category in $A_{1}, h$ can be made into a continous function from $a_{1}$ to $a_{2}$ satisfying (1)' for all $a_{1}$ in $A_{1}$.

\section{Bibliography}

1. N. Bourbaki, Eléments de Mathématique, Livre III, Topologie Generale, Chapters I, II, Paris, 1940.

2. — Eléments de Mathématique, Livre III, Topologie Generale, Chapter IX, Paris, 1948.

3. S. P. Diliberto, Pertubation of continuous surfaces, II, Technical Report No. 9, 
O.N.R. Research Contract Nonr-222(37), Dept. of Mathematics, University of California at Berkeley, 1957.

4. N. Dunford and J. T. Schwartz, Linear operators, vol. 1, Chapter 5, to appear.

5. W. H. Gottschalk and G. A. Hedlund, Topological dynamics, Amer. Math. Soc. Colloquium Publications, New York, 1955.

6. G. A. Hedlund, A class of transformations of the plane, Proc. Cambridge Philos. Soc. vol. 51 (1955) pp. 554-564.

7. S. Kakutani, On the continuity of eigenfunctions of minimal dynamical systems, to appear.

8. K. Yosida and S. Kakutani, Operator theoretical treatment of Markoff's process and mean ergodic theorem, Ann. of Math. vol. 42 (1941) pp. 188-228.

YALE UNIVERSITY

\section{ON SPACES WHICH ARE NOT OF COUNTABLE CHARACTER}

J. M. MARR

It is well known that the unit interval $I$ has a countable base and the fixed point property. By considering the maps $g(x)=x^{2}$ and $h(x)$ $=1-x$, one sees that there is no $x \in I$ such that for every continuous map $f: I \rightarrow I, x \in f(I)$ implies $f(x)=x$.

In Theorem 1 , it is shown that if $A$ is a closed, non-null proper subset of a locally connected, compact Hausdorff space $X$ which has a countable base, then there exists a continuous map $f: X \rightarrow X$ such that $A \cap f(X)$ is not contained in $A \cap f(A)$. Theorem 2 shows that certain nondegenerate topological spaces $X$ contain proper subsets $M$ such that for every continuous map $f: X \rightarrow X, M \cap f(X) \subset M \cap f(M)$. That is, for each of these spaces $X$ and every continuous map $f: X \rightarrow X, x \in M \cap f(X)$ implies $f^{-1}(x) \cap M \neq \varnothing$. The corollary is of interest in that, if $X$ satisfies the hypotheses of Theorem 2 and $M$ consists of a single point, then a fixed point of some of the maps $f: X \rightarrow X$ is located.

Theorem 1. Suppose $X$ is a connected, locally connected, compact Hausdorff space which has a countable base. If $A$ is any non-null, closed, proper subset of $X$, then there exists a continuous map $f: X \rightarrow X$ such that $A \cap f(X) \backslash A \cap f(A) \neq \varnothing$.

Proof. Since $X$ is compact Hausdorff and has a countable base, $X$ is metrizable. Hence $X$ is arcwise connected. Let $y \in X \backslash A$. Since

Received by the editors December 16, 1957 and, in revised form, April 3, 1958. 